Revista de Matemática: Teoría y Aplicaciones 1(1): 73-86 (1994)

\title{
FUNCIONES PERIÓDICAS, CUASIPERIÓDICAS Y CLASIFICACIÓN DE FUNCIONES
}

\author{
Edwin Castro Fernández ${ }^{1}$
}

\begin{abstract}
Resumen
En este trabajo presentamos algunos conjuntos significativos de funciones. Siguiendo las ideas de [2] introducimos las funciones cuasiperiódicas y sus propiedades elementales. Basados en [12] damos una clasificación de los conjuntos.
\end{abstract}

\begin{abstract}
In this work we present some significative sets of functions. Following the ideas of [2] we introduce the almost periodic functions and its elementary properties. Based in [12] we give a classification of the sets.
\end{abstract}

\section{Introducción}

En el presente trabajo vamos a estudiar algunos conjuntos de funciones continuas de $\mathbb{R}$ en $\mathbb{R}$ vamos a ultilizar la siguiente notación: $C$ el conjunto de funciones continuas de $\mathbb{R}$ en $\mathbb{R} ; B$ el conjunto de funciones continuas y acotadas de $\mathbb{R}$ en $\mathbb{R} ; C . U$ el conjunto de funciones uniformemente continuas de $\mathbb{R}$ en $\mathbb{R}$.

En los párrafos que siguen introduciremos también los conjuntos de funciones $P$ (periódicas y continuas) C.P (cuasiperiódicas). Aunque el conjunto $P$ es bien conocido, nosotros explotaremos algunas propiedades que nos servirán para introducir el conjunto C.P.

\section{El conjunto de funciones $C([1],[8])$}

El conjunto de funciones continuas de $\mathbb{R}$ en $\mathbb{R}$ y denotado con $C$ es bien conocido, este conjunto se puede dotar con la estructura de espacio vectorial definiendo la suma y la multiplicación escalar en forma usual; definiendo la multiplicación de funciones también en forma usual se tiene que $C$ es una álgebra.

\footnotetext{
${ }^{1}$ Escuela de Matemática, Universidad de Costa Rica
} 


\section{El conjunto de funciones C.U ([1],[8],[10])}

Sea $D \subset \mathbb{R}$ y $f: D \longrightarrow \mathbb{R}$ aplicación decimos que $f$ es uniformemente continua en $D$ si para todo $\varepsilon>0$ existe $\delta(\varepsilon)>0$ tal que: para todo $x, y \in D$ con $|x-y|<\delta(\varepsilon)$ se tiene que $|f(x)-f(y)|<\varepsilon$.

Denotaremos con C.U al conjunto de funciones uniformemente continuas de $\mathbb{R}$ en $\mathbb{R}$.

Es claro que si $f, g \in C . U$ entonces $f+g \in C . U$ y si $\lambda \in \mathbb{R}$ entonces $\lambda f \in C . U$, sin embargo no siempre el producto de dos funciones de C.U es una función de $C . U$, si consideramos por ejemplo la función $f(x)=x$ se tiene que $f \in C$.U, pero $g(x)=$ $f(x) f(x)=x^{2}$ no es una función de $C . U$. El resultado siguiente es bien conocido.

Proposición 3.1. Sean $a, b \in \mathbb{R}, \quad a<b$ y $f[a, b] \longrightarrow \mathbb{R}$ continua entonces $f$ es uniformemente continua.

Para la prueba de la proposición 3.1. véase ([8]).

\section{El conjunto de funciones $B$}

Decimos que una función $f: \mathbb{R} \longrightarrow \mathbb{R}$ es acotada si existe $M \in \mathbb{R}, \quad M>0$ tal que:

$$
|f(x)| \leq M \quad \forall x \in \mathbb{R}
$$

Si $f$ es una función acotada y continua diremos que $f \in B$. Es claro que si $f, g \in$ $B, \lambda \in \mathbb{R}$ entonces $\lambda f+g \in B$ y $f \cdot g \in B$. También se tiene que si $f \in C . U, g \in B$ entonces $f g \in C . U$, pero no es cierto que $B \subset C . U$ ([1]).

\section{El conjunto de funciones $P([2],[9],[10])$}

El conjunto $P$ es esencial en el presente trabajo, por eso presentaremos y probaremos algunas de sus propiedades vamos a decir que una función $f: \mathbb{R} \longrightarrow \mathbb{R}$ pertenece a $P$ si:

a) $f \in C$

b) $f$ es periódica, es decir: existe $T \in \mathbb{R}, T \neq 0$ tal que: $f(x+T)=F(x) \forall x \in \mathbb{R}$.

La propiedad (b) nos permite decir que $f$ es periódica de período $T$.

Fácilmente se tiene:

Lema 5.1. Si $f \in P$ entonces:

(a) $f$ es acotada $(f \in B)$.

(b) Denotando con $T$ un período de $f$ tenemos que:

i) $f$ es de período $-T$,

ii) $f$ es de período $m T \quad \forall m \in \mathbb{Z}$. 
El lema 5.1 nos permite trabajar solamente con períodos positivos.

Vamos a ver a continuación que la suma y el producto de dos funciones de $P$ no necesariamente es una función de $P$. En efecto, considere $g(x)=\cos t+\cos \sqrt{2} t$ se ve que cada uno de los miembros de la suma es una función de $P$, sin embargo si $g(x)$ fuera una función de $P$ se tendría que existiría un $T>0$ tal que: $\cos (t+T)+\cos \sqrt{2}(t+T)=$ $\cos t+\cos \sqrt{2} t \quad \forall t \in \mathbb{R}$ y en particular para $t=0$ tendremos: $\cos T+\cos \sqrt{2} T=2$ de donde $T=2 m \pi$ y $\sqrt{2} T=2 n \pi$ para algún $m, n \in \mathbb{Z} \backslash 0$, tendríamos entonces que: $\sqrt{2}=\frac{n}{m}$ lo cual es absurdo. Por lo tanto $g \notin P$.

Observe el lector que la suma $\cos t+\cos \sqrt{2} t$ se puede escribir en la forma:

$$
2 \cos \frac{\sqrt{2}-1}{2} t \cos \frac{\sqrt{2}+1}{2} t
$$

Se ve entonces que le producto de dos funciones de $P$ no siempre es una función de $P$. Cabe preguntarnos sin embargo: ¿En qué casos la suma de dos funciones de $P$ es una función de $P$ ?

Observe el lector en primer lugar que existen funciones periódicas que no pertenecen a $P$, un ejemplo es la función $f(x)=x-[x]$ que es periódica de período 1 pero no es continua.

Proposición 5.2 Sean $f, g$ funciones periódicas con períodos $T_{1}$ y $T_{2}$ respectivamente si $T_{1} / T_{2} \in \mathbb{Q}$ entonces $f+g$ es periódica.

Demostración. Podemos escribir $T_{1} / T_{2}=m / n$, con $m, n \in \mathbb{N}$, entonces $n T_{1}=m T_{2}$ y $T=n T_{1}=m T_{2}$ se tiene que:

$$
(f+g)(t+T)=f(t+T)+g(t+T)=f\left(t+n T_{1}\right)+g\left(t+m T_{2}\right)=f(t)+g(t) \quad \forall t \in \mathbb{R}
$$

Así $f+g$ es periódica de período $T$.

Surge ahora la pregunta ¿qué sucede si $f$ y $g$ son periódicas de períodos $T_{1}$ y $T_{2}$ respectivamente y $T_{1} / T_{2} \notin Q$ ? Considere tres números reales $a, b, c$ inconmensurables entre sí (es decir que ninguno de los cocientes $\frac{a}{b}, \frac{b}{c}, \frac{a}{c}$ son racionales) y definimos:

$$
\begin{aligned}
& f(x)=\sum_{m, n=-\infty}^{+\infty} \varphi(x-m a-n b)-\sum_{m, n=-\infty}^{+\infty} \varphi(x-m a-n c) \\
& g(x)=\sum_{m, n=-\infty}^{+\infty} \varphi(x-m b-n c)-\sum_{m, n=-\infty}^{+\infty} \varphi(x-m a-n b)
\end{aligned}
$$

donde

$$
\varphi(t)= \begin{cases}0 & \text { si } t \in \mathbb{R} \backslash\{0\} \\ 1 & \text { si } t=0\end{cases}
$$

Sea $x_{0} \in \mathbb{R}$ si $x_{0}=m a+n b$ para algún $m, n \in \mathbb{Z}$ ntonces $\quad \varphi\left(x_{0}-m a-n b\right)=1$. Se concluye que cualquiera de las dos series anteriores es convergente por la definición de $\varphi$, además se tiene: 


$$
f(x+a)=\sum_{m, n=-\infty}^{+\infty} \varphi(x-(m-1) a-n b)-\sum_{m, n=-\infty}^{+\infty} \varphi(x-(m-1) a-n c)=f(x)
$$

Análogamente: $g(x+b)=g(x)(f+g)(x+c)=(f+g)(x)$

Por lo tanto hemos construido dos funciones $f, g$ con períodos incomensurables cuya suma es periódica y su período es incomensurable con el período de $f$ y de $g$.

Los resultados que siguen serán útiles en el estudio de las funciones periódicas.

Proposición 5.3 Sea $x \in \mathbb{R} \backslash \prec$ Qentonces el conjunto: $A=\{m x+n: m, n \in \mathbb{Z}\}$ es denso en $\mathbb{R}$.

La demostración de esta proposición será incluida en el apéndice del presente trabajo.

Corolario 5.4. Sean $T_{1}, T_{2} \in \mathbb{R}$ tales que $T_{1} / T_{2} \notin Q$ entonces el conjunto

$$
S=\left\{m T_{1}+n T_{2}: m, n \in \mathbb{Z}\right\}
$$

es denso en $\mathbb{R}$.

Demostración. Sea $T_{1} / T_{2}=x$ entonces el conjunto: $A=\{m x+n: m, n \in \mathbb{Z}\}$ es denso en $\mathbb{R}$ lo cual implica que $T_{2} A$ es denso en $\mathbb{R}$ pero $T_{2} A=S$.

Observación. Si $T_{1} / T_{2} \notin Q$ entonces cada elemento del conjunto $S$ definido en el Corolario 5.4 se escribe de manera única, es decir si: $m T_{1}+n T_{2}=m^{\prime} T_{1}+n^{\prime} T_{2}$ con $m, n, m^{\prime}, n^{\prime} \in \mathbb{Z}$ entonces $m=m^{\prime}, \quad n=n^{\prime}$.

Proposición 5.5. Sea $f: \mathbb{R} \longrightarrow \mathbb{R}$ continua (a la derecha o a la izquierda) con la propiedad de que existe una sucesión $\left(a_{n}\right)_{n \in \mathbb{N}}$ no estacionaria y convergente tal que: $f\left(x+a_{n}\right)=f(x) \forall x \in \mathbb{R}, \quad n \geq 1$ entonces $f$ es constante.

La demostración de esta proposición será presentada en el apéndice.

Teorema 5.6. Sea $f \in P, f$ no constante entonces el conjunto de los períodos de $f$ es numerable.

Demostración. Sea $A=\{T>0: f(t+T)=f(t) \forall t \in \mathbb{R}\}$ y $A_{i}=A \cap[0, i], \quad i \geq 1$. Si $A_{1}$ fuera infinito tendría un punto de acumulación $T^{*}$ y entonces existiría una sucesión $\left(T_{n}\right)_{n \in \mathbb{N}}$ de elementos diferentes de $A_{i}$ convergente a $T^{*}$ por la proposición 5.5 se concluiría que $f$ sería constante, como $A_{i}$ es finito y $A=\bigcup_{i \in \mathbb{N}} A_{i}$ se deduce que $A$ es numerable.

Colorario 5.7. Si $f \in P$ entonces el conjunto de períodos de $f$ es un conjunto aislado. Este Corolario deduce de la prueba del teorema 5.6.

Colorario 5.8. Sea $f \in P$ no constante entonces $f$ posee un período positivo más pequeño.

Demostración. Sea $A=\{T>0: f(t+T)=f(t) \forall t \in \mathbb{R}\}$ y $T^{*}=\inf A$.

i) Si $T^{*} \in A$ entonces $T^{*}$ tiene la propiedad del enunciado.

ii) Si $T^{*} \notin A$ entonces $T^{*} \in A^{\prime}$ y existe una sucesión $\left(a_{n}\right)_{n \in \mathbb{N}}$ de elementos diferentes de $A$ tales que $a_{n} \longrightarrow T^{*}$. Resulta que $f$ sería constante, lo cual es absurdo.

Proposición 5.9. Sea $f \in P$. Si $f$ tiene dos períodos incomensurables entonces $f$ es constante. 
Demostración. Sean $T_{1}, T_{2}$ dos períodos incomensurables de $f$ como:

$$
S=\left\{m T_{1}+n T_{2}: m, n \in \mathbb{Z}\right\}
$$

es denso en $\mathbb{R}$ se tiene de la relación:

$$
f\left(m T_{1}+n T_{2}\right)=f(0) \forall m, n \in \mathbb{Z}
$$

y se deduce que

$$
f(y)=f(0) \forall y \in \mathbb{R}
$$

Proposición 5.10. Sean $f, g \in P, f, g$ no constantes, $f$ con período $T_{1}, g$ con período $T_{2}, f+g$ con período $T$. Si $T_{1}$ y $T_{2}$ son incomensurables entonces $T$ y $T_{1}, T$ y $T_{2}$ son también incomensurables.

Demostración. Supóngase que $T / T_{2} \in \mathbb{Q}$ entonces: $T / T_{2}=p / q$ y entonces que:

$$
(f+g)(t)=(f+g)(t+T)=(f+g)(t+q T)=f(t+q T)+g\left(t+p T_{2}\right)=f(t+q T)+g(t)
$$

de donde $f(t)=f(t+q T)$, se concluye que $f$ es periódica de período $q T$ y entonces es periódica de período $p T_{2}$. Como $f$ tiene período $p T_{2}$ y $T_{1}$ y ambos son incomensurables entonces $f$ es constante. Contradicción.

Proposición 5.11. Sean $T_{1}, T_{2} \in \mathbb{R}$ tales que $T_{1} / T_{2} \notin Q$ y sea

$$
A=\left\{m T_{1}+n T_{2}: m, n \in \mathbb{Z}\right\} .
$$

Sea $x \notin A$ entonces:

(1) Existen dos sucesiones de enteros $\left(a_{n}\right)_{n \in \mathbb{N}},\left(b_{n}\right)_{n \in \mathbb{N}}$ tal que $a_{n} T_{1}+b_{n} T_{2} \longrightarrow x$.

(2) Las sucesiones $\left(a_{n}\right)_{n \in \mathbb{N}},\left(b_{n}\right)_{n \in \mathbb{N}}$ son no acotadas.

(3) Algunas de las sucesiones $\left(a_{n}\right)_{n \in \mathbb{N}},\left(b_{n}\right)_{n \in \mathbb{N}}$ se puede elegir convergiendo a $+\infty$.

(4) Algunas de las sucesiones $\left(a_{n}\right)_{n \in \mathbb{N}}$, ó $\left(b_{n}\right)_{n \in \mathbb{N}}$ se puede elegir en $\mathbb{N}$ y la otra compuesta de enteros negativos.

La demostración de esta proposición aparecerá en el apéndice.

Proposición 5.12. Sea $f \in P$ no constante con período $T_{2}$ y sea $T \in \mathbb{R}$ tal que $T / T_{1} \notin Q$ entonces los límites:

$$
\lim _{n \longrightarrow+\infty} f(n T), \quad \lim _{n \longrightarrow-\infty} f(n T)
$$

no existen.

Demostración. Supóngase que $\lim f(n T)=l ; l \neq \pm \infty$ pues $f \in B$; existe $\alpha$ y $\beta$ suficientemente cerca tales que: $f(n T) \in[\alpha, \beta] \forall n \geq N$. Sea $x \in \mathbb{R}$ tal que:

i) $f(x) \notin[\alpha, \beta]$

ii) $f(-x) \notin[\alpha, \beta]$ 
iii) $x \neq m T_{1}+n T \quad \forall m, n \in \mathbb{Z}$

Como se ve, i), ii) se aseguran tomando $\alpha, \beta$ suficientemente cerca, iii) es claro.

Se tiene que existe una sicesión $\left(a_{n} T_{1}+b_{n} T\right)_{n \in \mathbb{N}}$ convergiendo a $x$. Pueden haber dos casos: (proposición 5.11)

1) $\left(a_{n}\right)_{n \in \mathbb{N}} \subset \mathbb{N},\left(b_{n}\right)_{n \in \mathbb{N}} \subset \mathbb{N}^{-}$.

2) $\left(a_{n}\right)_{n \in \mathbb{N}} \subset \mathbb{N}^{-},\left(b_{n}\right)_{n \in \mathbb{N}} \subset \mathbb{N}$

si se da el primer caso se considera la sucesión: $\left(-a_{n} T_{1}-b_{n} T\right)_{n \in \mathbb{N}}$ y se reduce al segundo. Consideramos el segundo caso, se tiene que:

$$
\alpha \leq f\left(a_{n} T_{1}+b_{n} T\right) \leq \beta \quad \forall n \geq N
$$

y tomando límite se deduce que: $f(x) \in[\alpha, \beta]$, lo cual es contradictorio.

Observaciones:

(1) La proposición anterior es cierta así: si $\left(a_{n}\right) \subset \mathbb{Z}$ y $a_{n} \longrightarrow+\infty$ o $a_{n} \longrightarrow-\infty$ entonces $\lim f\left(a_{n} T\right)$ no existe.

(2) Es fácil probar que si $f \in P$ y $f$ no es constante entonces $\lim _{x \longrightarrow+\infty} f(x)$ no existe. Aún sin la hipótesis de continuidad para $f$. Además si $\operatorname{lím}_{x \longrightarrow+\infty} f(x)$ existe entonces $f$ es constante.

(3) La proposición 5.12 es cierta también si $T / T_{1} \in Q$ y $T$ no es período de $f$ siempre que $f(T) \neq f(0)$. Como $\frac{T}{T_{1}}=\frac{p}{q}$ entonces $q T=p T_{1}$ se tiene entonces que:

$$
f(q T)=f\left(p T_{1}\right)=f(0), \quad f(n q T)=f\left(n p T_{1}\right)=f(0), \quad f((n q+1) T)=f(T) .
$$

Así el límite $\operatorname{lím}_{n \longrightarrow+\infty} f(n T)$ no existe.

Proposición 5.13. Sea $f, g \in P, f$ con período $T_{1}, g$ con período $T_{2}, T_{1} / T_{2} \notin \mathbb{Q}, f \mathrm{y}$ $g$ no constantes. Entonces $f+g$ no puede ser periódica.

Demostración. Supóngase que $f+g$ es periódica de período $T$.

(i) $(f+g)\left(a T_{1}+b T\right)=f(0)+g(a T) \quad \forall a, b \in \mathbb{Z}$

(ii) $(f+g)\left(c T_{2}+b T\right)=f\left(c T_{2}\right)+g(0) \forall c, b \in \mathbb{Z}$

Supóngase que existe una sucesión $a_{n} T_{1}+b_{n} T \longrightarrow x$ con $\left(a_{n}\right)_{n \in \mathbb{N}} \subset \mathbb{N}$ y $a_{n} \longrightarrow+\infty$ y además $x \neq m T_{1}+n T \quad \forall m, n \in \mathbb{Z}$. Considerando (i):

$$
(f+g)\left(a_{n} T_{1}+b_{n} T\right)=f(0)+g\left(a_{n} T_{1}\right)
$$

y haciendo tender $n \longrightarrow+\infty$, el miembro izquierdo tiende a $(f+g)(x)$ y el derecho no tiene límite cuando $n \longrightarrow+\infty$. 


\section{Otras propiedades de las funciones periódicas}

Proposición 6.1. Si $f \in P$ entonces $f \in C . U$. ([8],[10]).

Demostración. Sea $f: \mathbb{R} \longrightarrow \mathbb{R}$ periódica y continua y $T>0$ período de $f$, como $f$ es continua en $[0,3 T]$ entonces $f$ es uniformemente continua así: para todo $\varepsilon>0$ existe $\delta(\varepsilon)>0$ tal que $\delta(\varepsilon)<T$ y con la propiedad de que si $x, y \in[0,3 T]$ con $|x-y|<\delta(\varepsilon)$ entonces $|f(x)-f(y)|<\varepsilon$.

Vamos a probar que tiene lugar la implicación $|x-y|<\delta$ implica $|f(x)-f(y)|<\varepsilon$ sin la restricción $x, y \in[0,3 T]$.

Sean $x, y \in \mathbb{R}$ tales que $|x-y|<\delta$ se tiene que: $0 \leq x\left[\frac{x}{T}\right] T<T$ y entonces $x^{\prime}=$ $x+T-\left[\frac{x}{T}\right] T \in[T, 2 T], y^{\prime}=y+T-\left[\frac{x}{T}\right] T \in[0,3 T]$ además $\left|x^{\prime}-y^{\prime}\right|=|x-y|<\delta$ y entonces $\left|f\left(x^{\prime}\right)-f\left(y^{\prime}\right)\right|<\varepsilon$ pero $f(x)-f(y)|=| f\left(x^{\prime}\right)-f\left(y^{\prime}\right) \mid$, y se sigue el resultado.

A continuación nos dedicaremos a estudiar la composición de funciones periódicas.

Es claro que si $f: \mathbb{R} \longrightarrow \mathbb{R}$ y $g: \mathbb{R} \longrightarrow \mathbb{R}$ periódica entonces $f \circ g$ es periódica.

Si $g \in P, \quad f \in C$ no necesariamente se tiene que $g \circ f$ es periódica, por ejemplo si $f(x)=x^{2}, g(x)=\operatorname{sen} x$ fácilmente se ve que $(g \circ f)(x)=\operatorname{sen} x^{2}$ no es periódica.

Sin embargo si $f \in P$ y $p(x)=a x+b$ se tiene que $\varphi(x)=(f \circ p)(x)=f(a x+b)$ es periódica.

El siguiente resultado responde parcialmente a las inquietudes anteriores.

Teorema 6.2. Sean $f, g \in C$ tales que:

1) $f \in P, f$ no constante.

2) $\lim _{x \longrightarrow+\infty} \frac{g(x)}{x}=+\infty$ entonces $f \circ g \notin P$.

Demostración. Sea $T>0$ período de $f$ entonces $f$ alcanza en $[0, T]$ su máximo y su mínimo $f(u)=m=\min \{f(x): x \in[0, T]\}, f(v)=M=\max \{f(x): x \in[0, T]\}$. Sea $\varepsilon=\frac{M-m}{2}>0$ entonces existe $\delta>0$ tal que:

$$
|x-y|<\delta \text { implica }|f(g(x))-f(g(y))|<\frac{M-m}{2}
$$

Por otra parte existe un intervalo de longitud $\delta$ en el cual $g$ tiene una variación mayor que $T$. En efecto, si $|x-y|<\delta$ entonces $|g(x)-g(y)| \leq T$ entonces:

$$
\left|\frac{g(n \delta)}{n \delta}\right| \leq \frac{|g(0)|+|g(\delta)-g(0)|+\ldots+|g(n \delta)-g((n-1) \delta)|}{n \delta} \leq \frac{|g(0)|}{n \delta}+\frac{T}{\delta}
$$

y entonces

$$
\lim _{n \longrightarrow+\infty}\left|\frac{g(n \delta)}{n \delta}\right| \leq \frac{T}{\delta}
$$

lo cual contradice 2$)$.

Sea $\left[n_{0} \delta,\left(n_{0}+1\right) \delta\right]$ con la propiedad de que existen $x_{1}, x_{2} \in\left[n_{0} \delta,\left(n_{0}+1\right) \delta\right]$ tal que: $\left|g\left(x_{1}\right)-g\left(x_{2}\right)\right|>T$ entonces entre $x_{1}, x_{2}$ existen $t_{1}, t_{2}$ tales que $f\left(g\left(t_{1}\right)\right)=f(u)=m$, 
$f\left(g\left(t_{2}\right)\right)=f(v)=M$ así pues $\left|t_{1}-t_{2}\right|<\delta$ y $\left|f\left(g\left(t_{1}\right)\right)-f\left(g\left(t_{2}\right)\right)\right|=M-m$ lo cual contradice (1) por lo tanto $f \circ g \notin P$.

Observación. Si en lugar de la hipótesis 2) escribimos $\lim _{x \longrightarrow+\infty} \frac{g(x)}{x}=-\infty$ el resultado sigue con la misma demostración.

Colorario 6.3. Sea $f \in P$ no constante y $p$ polinomio entonces $f \circ p \in P$ sí y solo sí el grado de $p$ es $\leq 1$.

Colorario 6.4. Sea $g \in P, \quad f: \mathbb{R} \longrightarrow \mathbb{R}$ derivable y de clase $C^{1}$ tal que $\lim _{x \longrightarrow+\infty} f(x)=$ $\lim _{x \longrightarrow+\infty} f^{\prime}(x)=\infty$, entonces $g \circ f \notin P$.

\section{Funciones cuasiperiódicas $([3],[4],[5],[6],[7],[9])$}

Consideremos la ecuación diferencial

$$
y^{(4)}+3 y^{\prime \prime}+2 y=0
$$

cuya solución de clase $C^{1}$ es:

$$
y(t)=A \cos t+B \operatorname{sen} T+C \cos \sqrt{2} t+D \operatorname{sen} \sqrt{2} t \quad A, B, C, D \in \mathbb{R}
$$

Cada uno de los términos de la solución es una función periódica, sin embargo ya vimos anteriormente que la aplicación $t \longrightarrow \cos t+\cos \sqrt{2} t$ no es periódica y sin embargo es solución de la ecuación diferencial.

Lo anterior motivó a Bohr a introducir una clase de funciones más amplia llamada funciones cuasiperiódicas y denotadas con C.P.

Una función $f: \mathbb{R} \longrightarrow \mathbb{R}$ se dice cuasiperiódica si:

a) $f \in C$

b) $\forall \varepsilon>0$ existe $l>0$ tal que para todo $a \in \mathbb{R}$ existe: $\tau \in[a, a+l]$ con la propiedad

$$
|f(t+\tau)-f(t)|<\varepsilon \forall t \in \mathbb{R}
$$

Proposición 7.1. $P \subset C . P$.

Demostración. Sea $f \in P$ por hipótesis existe $T \in \mathbb{R}^{+}$tal que $f(t+T)=f(t) \forall t \in$ $\mathbb{R}$.

Sea $\varepsilon>0$ y $l=T>0$. Sea $a \in \mathbb{R} \quad \tau=n T$ con $n=1+[a / T]$ se tiene que: $a \leq \tau \leq a+l$ puesto que da

$$
\frac{a}{T}-1<\left[\frac{a}{T}\right] \leq \frac{a}{T} \text { ó } \frac{a}{T}-1<n-1 \leq \frac{a}{T},
$$

se tiene que $a<n T \leq a+T$ y se obtiene $a \leq \tau \leq a+l$.

Sea $t \in \mathbb{R}$, se tiene que:

$$
|f(t+\tau)-f(t)|=|f(t+n T)-f(t)|=|f(t+T)-f(t)|=0
$$

Proposición 7.2. C.P $\subset B$. 
Demostración. Sea $f \in C . P$ y sea $\varepsilon=1$ entonces existe $l>0$ tal que para cualquier $a \in \mathbb{R}$ se puede hallar $\tau \in[a, a+l]$ con $|f(t+\tau)-f(t)|<1 \quad \forall t \in \mathbb{R}$. Como $f$ es continua $f$ es acotado en $[0, l]$ y entonces existe $L>0$ tal que $|f(t)| \leq L \forall t \in[0, l]$. Sea $t \in \mathbb{R}$ para $a=-t$ existe $\tau \in[-t,-t+l]$ tal que:

$$
|f(t)| \leq|f(t)-f(t+\tau)|+|f(t+\tau)|<1+L
$$

Proposición 7.3. C.P C C.U

Demostración. Sea $f \in C . P$ y $\varepsilon>0$ entonces existe $l>0$ tal que para todo $a \in \mathbb{R}$ existe $\tau \in[a, a+l]$ con: $|f(s+\tau)-f(s)|<\varepsilon \forall s \in \mathbb{R}$

Como $f$ es uniformemente continua en $[-1,1+l]$ entonces existe $\delta>0$ con $\delta \leq 1$ tal que para cualquier $s^{\prime}, s^{\prime \prime}$ con $s^{\prime}, s^{\prime \prime} \in[-1,1+t]$ con $\left|s^{\prime}-s^{\prime \prime}\right| \leq \delta$ tenemos que $\left|f\left(s^{\prime}\right)-f\left(s^{\prime \prime}\right)\right|<\varepsilon$.

Sea ahora $t^{\prime}, t^{\prime \prime} \in \mathbb{R}$ con $\left|t^{\prime}-t^{\prime \prime}\right|<\delta$ poniendo $a=-t^{\prime}$ existe $\tau \in\left[-t^{\prime},-t^{\prime}+1\right]$ tal que $|f(s+t)-f(s)|<\varepsilon \forall s \in \mathbb{R}$.

Como $t^{\prime}+\tau \in[0, l] \subset[-1,1+l]$ y $t^{\prime \prime}+\tau \in[-\delta, \delta+l] \subset[-1,1+l]$ se deduce que:

$$
\left|f\left(t^{\prime}\right)-f\left(t^{\prime \prime}\right)\right| \leq\left|f\left(t^{\prime}\right)-f\left(t^{\prime}+\tau\right)\right|+\left|f\left(t^{\prime}+\tau\right)-f\left(t^{\prime \prime}+\tau\right)\right|+\left|f\left(t^{\prime \prime}+\tau\right)-f\left(t^{\prime \prime}\right)\right|<3 \varepsilon
$$

Proposición 7.4. Sea $f \in C$ monótona y no constante entonces $f \notin C . P$.

Demostración. Existen $a, b \in \mathbb{R}, \quad a<b$ tales que $f(a)<f(b)$ (ó $f(a)>f(b)$ ). Supóngase que $f$ es creciente. Supóngase que $f$ es cuasiperiódica. Sea $\varepsilon=f(b)-f(a)>0$ entonces existe $l>0$ y existe $\tau \in[b, b+l]$ tal que: $|f(t+\tau)-f(t)|<\varepsilon \forall t \in \mathbb{R}$.

Se tiene en particular que:

$$
f(a+\tau)-f(a)<\varepsilon \text { pero } f(a+\tau)-f(a) \geq f(b)-f(a)
$$

lo cual es contradictorio. Se sigue el resultado.

Proposición 7.5. Sea $f \in C . P$ entonces $\operatorname{lím}_{x \longrightarrow+\infty} f(x)$ existe si y solo si $f$ es constante.

Demostración. Supóngase que $f \in C . P$ y que el límite existe, sin pérdida de generalidad supóngase que el límite es igual a cero.

Vamos a suponer que $f$ no es constante. Sea $x_{0} \in \mathbb{R}$ tal que $f\left(x_{0}\right)>\delta>0$ (análogamente si existe $x_{0} \in \mathbb{R}$ con $f\left(x_{0}\right)<\delta<0$. Probaremos que dado cualquier $M>0$ existe $x_{1} \in \mathbb{R}$ tal que $f\left(x_{1}\right)>\delta>0$ con $x_{2}>M$.

Sea $\varepsilon>0$ tal que $f\left(x_{0}\right)-\varepsilon>\delta$, existe $l>0$ tal que para todo $a \in \mathbb{R}$ existe $\tau \in[a, a+l]$ y:

$$
\left|f\left(x_{0}+\tau\right)-f\left(x_{0}\right)\right|<\varepsilon
$$

se sigue que

$$
f\left(x_{0}+\tau\right)>f\left(x_{0}\right)-\varepsilon
$$

Sea $a$ de tal forma que $x_{0}+a>M$ entonces podemos elegir $\tau$ con $\tau \in[a, a+l]$ para que se cumpla (2) y se tiene: $x_{0}+\tau>x_{0}+a$ y por (2) $f\left(x_{0}+\tau\right)>\delta>0$. Si se toma $x_{1}=x_{0}+\tau$ se tiene el resultado.

Proposición 7.6. Sea $f \in C . P$. y $g \in C$ entonces $g \circ f \in C . P$.

Demostración. Como $f \in C . P$ entonces $A=\overline{f(\mathbb{R})}$ es compacto y entonces $g: A \longrightarrow$ $\mathbb{R}$ es uniformemente continua.

Sea $\varepsilon>0$ se tiene que: 
(a) Existe $\delta>0$ tal que $\forall x, y \in A$ con $|x-y|<\delta$ se tiene que $|g(x)-g(y)|<\varepsilon$.

(b) Existe $l>0$ tal que para todo $a \in \mathbb{R}$ existe $\tau \in[a, a+l]$ con: $|f(x+\tau)-f(x)|<$ $\delta \forall x \in \mathbb{R}$.

Combinando (a) y (b) tenemos que: existe $l>0$ tal que para todo $a \in \mathbb{R}$ existe $\tau \in[a, a+l]$ con $|g(f(x+\tau))-g(f(x))|<\varepsilon \forall x \in \mathbb{R}$, es decir $g \circ f \in C . P$.

Observación 1. Se $f \in C ; g \in P$ no se sigue $g \circ f \in C$.P. Sea por ejemplo $f(x)=x^{2}$, $g(x)=\operatorname{sen} x$ entonces $(g \circ f)(x)=\operatorname{sen} x^{2}$. Se tiene que $g \circ f \notin C . U$ entonces $g \circ f \notin C . P$. (§9 Ej. 3).

Observación 2. Vale la proposición 6.2 si en lugar de $P$ ponemos C.P.

Proposición 7.7. La suma de dos funciones cuasiperiódicas es una función cuasiperiódica.

Vamos a probar la proposición 7.7 en la forma que sigue:

Si $f_{1}, f_{2} \in C . P$ y $\varepsilon>0$ entonces existe $l>0$ tal que para todo $b \in \mathbb{R}$ existe $\tau \in[b, b+l]$ con:

$$
\left|f_{1}(t+\tau)-f_{1}(t)\right|<\varepsilon, \quad\left|f_{2}(t+\tau)-f_{2}(t)\right|<\varepsilon
$$

Demostración. (a) Sean $f_{1}, f_{2} \in C . P$ entonces existen $l_{1}, l_{2}>0$ tales que para todo $a \in \mathbb{R}$ existe $\tau_{i}(a) \in\left[a, a+l_{i}\right] \quad i=1,2$ con:

$$
\left|f_{i}\left(t+\tau_{i}(a)\right)-f_{i}(t)\right|<\varepsilon / 4 \quad \forall t \in \mathbb{R}
$$

Sea $l_{0}=\operatorname{Max}\left\{l_{1}, l_{2}\right\}$ como $f_{1}, f_{2}$ son uniformemente continuas entonces existe $\delta>0$, $\delta \leq l_{0}$ tal que para todo $s, t \in \mathbb{R}$ con $|t-s| \leq \delta$ se tiene:

$$
\left|f_{i}(t)-f_{i}(s)\right|<\frac{\varepsilon}{4}
$$

(b) Sea $\sigma_{i}(a)=p_{i} \delta i=1,2$, donde $p_{i}$ cumple:

$$
\left|\tau_{1}(a)-p_{i} \delta\right|=\inf \left\{\left|\tau_{i}(a)-q \delta\right|: q \in \mathbb{Z}, a \leq q \delta \leq a+l_{0}\right\}
$$

es claro que:

$$
\sigma_{i}(a) \in\left[a, a+l_{0}\right] \mathrm{y}\left|\tau_{i}(a)-\sigma_{i}(a)\right| \leq \delta
$$

lo cual nos da:

$$
\left|f_{i}\left(s+\sigma_{i}(a)\right)-f_{i}(s)\right|<\frac{\varepsilon}{2} \forall s \in \mathbb{R}
$$

en virtud de (3) y (4).

(c) Observe que:

$$
\left|\sigma_{1}(a)-\sigma_{2}(a)\right| \leq l_{0} .
$$

Sea $C=\left\{\left(\sigma_{1}(a), \sigma_{2}(a)\right): a \in \mathbb{R}\right.$. Introducimos en $C$ una relación de equivalencia definida así:

$$
\left(\sigma_{1}(a), \sigma_{2}(a)\right) \sim\left(\sigma_{1}(b), \sigma_{2}(b) \text { si y solo si }\left|\sigma_{1}(a)-\sigma_{2}(a)\right|=\left|\sigma_{1}(b)-\sigma_{2}(b)\right|\right.
$$


Obsérvese de (5) que el conjunto $C / \sim$ tiene un número finito de clases de equivalencia $\widehat{C}_{1}, \ldots, \widehat{C}_{n}$. En cada clase elegimos un representante $\left(\sigma_{1}\left(a_{k}\right), \sigma_{2}\left(a_{k}\right)\right) ; a_{k} \in \mathbb{R}$. Sea:

$$
l_{1}^{\prime}=\operatorname{Sup}\left\{\mid \sigma_{i}\left(a_{k}\right): i=1,2\right\}
$$

(d) Sea $l=l_{0}+2 l_{1}^{\prime}$. Sea $b \in \mathbb{R}$ y $a=b+l_{1}^{\prime}$.

Considere los números $\sigma_{1}(a), \sigma_{2}(a)$ de la etapa (b), por (c) existe $k \in\{1, \ldots, n\}$ tal que $\left(\sigma_{1}(a), \sigma_{2}(a)\right) \in \widehat{C}_{k}$ entonces

$$
\left|\sigma_{1}(a)-\sigma_{2}(a)\right|=\left|\sigma_{1}\left(a_{k}\right)-\sigma_{2}\left(a_{k}\right)\right|
$$

denotando con $\eta=\operatorname{Sign}\left(\sigma_{1}(a)-\sigma_{2}(a)\right) \operatorname{Sign}\left(\sigma_{1}\left(a_{k}\right)-\sigma_{2}\left(a_{k}\right)\right)(7)$ se escribe como:

$$
\sigma_{1}(a)-\eta \sigma_{1}\left(a_{k}\right)=\sigma_{2}(a)-\eta \sigma_{2}\left(a_{k}\right)
$$

Denotaremos con $\tau$ el valor común de las expresiones (8) y como:

$$
\sigma_{1}(a) \in\left[a, a+l_{1}\right], \quad \sigma_{2} \in\left[a, a+l_{2}\right] \text { y }\left[a, a+l_{i}\right] \subset\left[a, a+l_{0}\right]=\left[b+l_{1}^{\prime}, b+l_{1}^{\prime}+l_{0}\right] .
$$

Se tiene por (6) que $\tau \in\left[b, b+l_{0}+2 l_{1}^{\prime}\right]=[b, b+l]$.

Finalmente, sea $t \in \mathbb{R}$ se tiene que:

$f_{i}(t+\tau)-f_{i}(t)|\leq| f_{i}\left(t+\sigma_{i}(a)-\eta \sigma_{i}\left(a_{k}\right)\right)-f_{i}\left(t-\eta \sigma_{i}\left(a_{k}\right)\right)|+| f_{i}\left(t-\eta \sigma_{i}\left(a_{k}\right)\right)-f_{i}(t) \mid<\varepsilon \quad i=1,2$

Colorario 7.9. Si $c \in \mathbb{R}$ y $f, g \in C . P$ entonces $c f+g \in C . P$.

Proposición 7.10. Si $f \in C . P$ entonces $f^{2} \in C . P$

Demostración. Como $f \in C . P$ implica $f \in B$, entonces $|f(x)| \leq M \quad \forall x \in \mathbb{R}$ y además:

$$
\left|f^{2}(x+\tau)-f^{2}(x)\right|=|f(x+\tau)+f(x)||f(x+\tau)-f(x)| \leq M|f(x+\tau)-f(x)|
$$

se sigue el resultado.

Proposición 7.11. Si $f, g \in C . P$ entonces $f \cdot g \in C . P$

Demostración. El resultado se sigue de escribir:

$$
f \cdot g=\frac{1}{4}\left\{(f+g)^{2}-(f-g)^{2}\right\}
$$

Proposición 7.11. Si $f \in C . P$ y $\inf \{|f(x)|: x \in \mathbb{R}\}=m>0$ entonces $1 / f \in C . P$. Demostración.

$$
\left|\frac{1}{f(x+\tau)}-\frac{1}{f(x)}\right|=\left|\frac{f(x+\tau)-f(x)}{f(x+\tau) f(x)}\right| \leq \frac{|f(x+\tau)-f(x)|}{m^{2}}
$$

y se sigue el resultado.

Colorario 7.12. Si $f, g \in C . P$ y in $f\{|g(x)|: x \in \mathbb{R}\}=m>0$ entonces $f / g \in C . P$. (El Corolario 7.12 se sigue de las proposiciones 7.8 y 7.9 ). 


\section{Ejemplos}

1.) $f \in C \backslash(C . U \cup B) f(x)=x^{2}$

2.) $f \in C . U \backslash B \quad f(x)=x$

3.) $f \in B \backslash C . U \quad f(x)=\operatorname{sen} x^{2}$.

Prueba: Sea $\varepsilon=1 / 2$. Si $f$ fuera uniformemente continua existiría $\delta>0$ tal que $\forall x, y \in \mathbb{R},|x-y|<\delta$ se tiene $|f(x)-f(y)|<1 / 2$. Sea $n \in \mathbb{N}$ tal que

$$
|\sqrt{n \pi}-\sqrt{(2 n+1) \pi / 2}|<\delta
$$

entonces se va a tener

$$
\left.1=|0-1|=|\operatorname{senn} \pi-\operatorname{sen}(2 n+1) \pi / 2|=\mid \operatorname{sen}(\sqrt{n} \pi)^{2}-\operatorname{sen}(\sqrt{(2 n+1) \pi / 2}\}\right)^{2} \mid<\frac{1}{2}
$$

4.) $f \in B \cap C . U \backslash C . P$. Sea $f(x)=\operatorname{arctanx}$. En primer lugar $f \notin C . P$ (Proposición 7.5). Se tiene además que $f \in B$. Como

$$
|f(x)-f(y)|=|x-y|\left|f^{\prime}(\xi)\right|=|x-y| \frac{1}{1+\xi^{2}} \leq|x-y| \forall x, y \in \mathbb{R}, \quad \xi \in \overline{x y}
$$

se sigue que $f \in C . U$.

5.) $f \in C . P \backslash P . f(x)=\cos x+\cos \sqrt{2} x(\operatorname{ver} \S 5)$

6.) $f \in P . f(x)=\cos x$

\section{Diagrama de funciones}




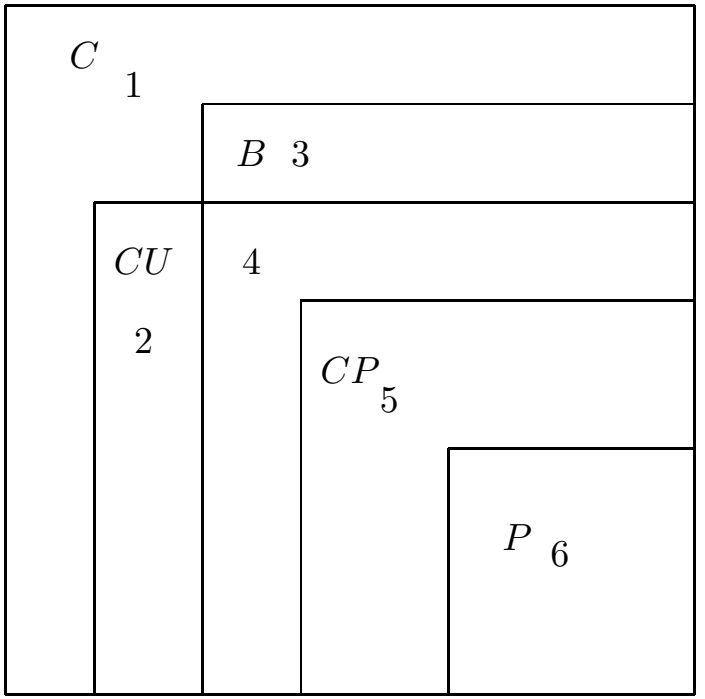

APENDICE

\section{Sobre un conjunto denso en $\mathbb{R}$}

Sea $x \in \mathbb{R} \backslash Q$ y $A=\{m x+n: m, n \in \mathbb{Z}\}$. Vamos a probar que $A$ es denso en $\mathbb{R}$. (Proposición 5.3)

Vamos a probar que para cualquier intervalo abierto y acotado $I \subset \mathbb{R} \backslash\{0\}$ tenemos que $I \cap A \neq \emptyset$. Se tiene evidentemente que existe $n \in \mathbb{Z}$ tal que $\left.\frac{1}{n} I \subset\right] 0,1[$ y tenemos que: $\frac{1}{n} I \cup A \neq \emptyset$ si y solo si $I \cap A \neq \emptyset$, por lo tanto podemos suponer que $\left.I \subset\right] 0,1[$.

Sea $I=] 0, \varepsilon[$ con $0<\varepsilon<1$ y consideremos $((x))=x-[x]$ sean $((x)),((2 x)), \ldots,((r x))$ donde $r \in \mathbb{N}$ y $\frac{1}{r}<\varepsilon$. Los números $((x)),((2 x)), \ldots,((r x))$ son distintos dos a dos pues $x \in I$.

Sean $p, q \in\{1, \ldots, r+1\}$ tales que:

$$
((p x))-((q x)) \leq \frac{1}{r}<\varepsilon
$$

Como $((p x))-((q x))=[q x]-[p x]+(p-q) x$, se deduce que $I \cap A \neq \emptyset$.

Sea ahora $I=] c, d[, \quad 0<c<d<1$. Considere $-I=]-d,-c[, 1-I=\{1-y: y \in$ $I\}=] 1-d, 1-c[$. Como $1-c>0$ entonces existe $1-p \in(1-I) \cap A$ con $P \in I$, de donde $1-p \in A$ y así $-p \in A-1=A$, o sea $p \in A$. Así $p \in A \cap I$. Se concluye que en cualquier caso se tiene $I \cap A \neq \emptyset$.

Proposición 5.5. Sea $f: \mathbb{R} \longrightarrow \mathbb{R}$ continua (a la derecha o a la izquierda) en $\mathbb{R}$ con la propiedad de que existe una sucesión $\left(a_{n}\right)_{n \geq 1}$ no estacionaria y convergente tal que: $f\left(x+a_{n}\right)=f(x) \forall x \in \mathbb{R}, \forall n \geq 1$, entonces $f$ es constante.

Demostración. Podemos suponer que $\lim a_{n}=0$. De la hipótesis deducimos que:

$$
\begin{gathered}
f\left(a_{n}\right)=f(0), \quad n \geq 1 \\
f\left(2 a_{n}\right)=f\left(a_{n}\right)=f(0)
\end{gathered}
$$


y en general: $f\left(m a_{n}\right)=f(0) \forall m, n \geq 1$.

Se deduce también que $f(0)=f\left(-a_{n}\right) n \geq 1$ y entonces: $f\left(-m a_{n}\right)=f(0) \forall m, n \geq 1$ y así se tiene que

$$
f\left(m a_{n}\right)=f(0) \forall n \in \mathbb{N}, m \in \mathbb{Z}
$$

Sea $x>0$, como $f$ es continua en $x$ se tiene que $f\left(x^{+}\right)=f(x)$. Para cada $k \geq 1$ sea $m_{k}$ el primer número natural tal que $x_{k}=m_{k}\left|a_{k}\right| \geq x$ y entonces:

$$
0 \geq x_{k}-x \geq\left|a_{k}\right| \text { y } f\left(m_{k} a_{k}\right)=f(0) \forall k \geq 1
$$

Como $a_{k} \longrightarrow 0$ tenemos $x_{k} \searrow x$ y entonces $f\left(x^{+}\right)=\lim f\left(x_{k}\right)=f(0)$ y como $f(x)=f\left(x^{+}\right)$se tiene que $f(x)=f(0)$.

En forma análoga se prueba que $f(x)=f(0) \forall x<0$.

Proposición 5.11. Sean $T_{1}, T_{2} \in \mathbb{R}$ tales que $T_{1} / T_{2} \notin \mathbb{Q}$. Supongamos que $x \notin A$ entonces:

(1) Existen dos sucesiones de enteros $\left(a_{n}\right)_{n \in \mathbb{N}},\left(b_{n}\right)_{n \in \mathbb{N}}$ tales que: $a_{n} T_{1}+b_{n} T_{2} \longrightarrow x$. La sucesión $\left(a_{n} T_{1}+b_{n} T_{2}\right)_{n \in \mathbb{N}}$ se puede elegir con todos los términos diferentes.

(2) Una de las sucesiones $\left(a_{n}\right)_{n \in \mathbb{N}}$ o $\left(b_{n}\right)_{n \in \mathbb{N}}$ se puede elegir tendiendo a $+\infty$ y la otra $-\infty$.

(3) Una de las sucesiones $\left(a_{n}\right)_{n \in \mathbb{N}}$ o $\left(b_{n}\right)_{n \in \mathbb{N}}$ se puede elegir de enteros positivos y la otra de enteros negativos.

\section{Demostración.}

(1) Evidente pues $\bar{A}=\mathbb{R}$.

(2) Supongamos que $\left(a_{n}\right)_{n \in \mathbb{N}}$ es acotada, entonces el conjunto $\left\{a_{1}, a_{1}, l d o t s, a_{n}, \ldots\right\}$ sería finito y se tendría que la sucesión $\left\{a_{1} T_{1}+b_{1} T_{2}, a_{2} T_{1}+b_{2} T_{2}, \ldots\right\}$ sería finito y entonces $x \in A$. Contradición. Por lo tanto ninguna de las sucesiones $\left(a_{n}\right)_{n \in \mathbb{N}}$ o $\left(b_{n}\right)_{n \in \mathbb{N}}$ es acotada.

Entonces alguna de las sucesiones tiene un número infinito de términos positivos supóngase que es $\left(a_{n}\right)_{n \in \mathbb{N}}$, considérese la sucesión $a_{n_{k}} \longrightarrow+\infty$ entonces la subsucesión $a_{n_{k}} T_{1}+b_{n_{k}} T_{2} \longrightarrow x$ cuando $k \longrightarrow+\infty$ y se debe tener que $b_{n_{k}} \longrightarrow-\infty$ cuando $k \longrightarrow-\infty$.

(3) Resulta fácil de (2).

\section{Referencias}

[1] Castro, Edwin; Sobre algunas funciones acotadas. (por aparecer).

[2] Cooke, Roger (1981) Almost Periodic Functions, Amer. Math. Monthly. 88(7), 515-525.

[3] Besicovitch, A.S. (1954) Almost Periodic Functions. Dover Publications Inc. New York.

[4] Bohr, Harold (1951) Almost Periodic Functions. Chelsea Publishing Company. New York. 
[5] Fink, A.M. (1977) Almost Periodic Differential Equations. Lecture Notes in Mathematics 377. Springer-Verlag.

[6] Titchmarsh, E.C. (1986) The theory of Functions. Second Editions. Oxford University Press. 1939. (corregida 1986).

[7] Muntean, Ioan (1990) Analizā Functionala: Capitale Speciale. Cluj. Napoca. Universitatea Babes, Bolyai.

[8] Arnaudiès, J.M. (1991) Cours de Mathematiques - 2 Analyse. Dunod Université. Paris.

[9] Barrantes, Delgado, Geiner (1973) Funciones cuasiperiódicas. Universidad de Costa Rica. Tesis presentada para optar el grado de Licenciado en Matemática.

[10] Dalyay Pal, Balogh Zoltam (1986) Functii Uniform Continue. Gazeta Matematică, Anul XCI, Nr11-12 (1986), 412-417.

[11] Niculescu, Liliana (1990) O Metoda de Calcul a Primitivelor xxxx Functii Periodice, Gazeta Matematică. Anul XCVI, Nr8, 281-284.

[12] Muntean, Ioan (1990) Clasificaci'on de Algunas Funciones Reales sobre un intervalo compacto, Ciencias Matemáticas, Volumen 1, número 1, 36-41. 